\title{
CAP1 is overexpressed in human epithelial ovarian cancer and promotes cell proliferation
}

\author{
MINHUI HUA ${ }^{1 *}$, SUJUAN YAN ${ }^{1,2 *}$, YAN DENG $^{1}$, QINGHUA XI ${ }^{1}$, RONG LIU $^{4}$, SHUYUN YANG $^{3}$, \\ JIAN LIU ${ }^{1}$, CHUNHUI TANG ${ }^{1}$, YINGYING WANG ${ }^{5}$ and JIANXIN ZHONG ${ }^{1}$ \\ ${ }^{1}$ Department of Obstetrics and Gynecology, Affiliated Hospital of Nantong University, Nantong, Jiangsu 226001; \\ ${ }^{2}$ Department of Obstetrics and Gynecology, The First People's Hospital of Jingmen, Jingmen, Hubei 448000; \\ Departments of ${ }^{3}$ Pathology and ${ }^{4}$ Oncology, Nantong University Cancer Hospital; ${ }^{5}$ Department of Pathogen Biology, \\ Medical College, Nantong University, Nantong, Jiangsu 226001, P.R. China
}

Received August 26, 2014; Accepted January 27, 2015

DOI: $10.3892 / \mathrm{ijmm} .2015 .2089$

\begin{abstract}
Adenylate cyclase-associated protein 1 (CAP1) regulates both actin filaments and the Ras/cAMP pathway in yeast, and has been found play a role in cell motility and in the development of certain types of cancer. In the present study, we investigated CAP1 gene expression in human epithelial ovarian cancer (EOC). Western blot analysis and immunohistochemistry were performed using EOC tissue samples and the results revealed that CAP1 expression increased with the increasing grade of EOC. In the normal ovarian tissue samples however, CAP1 expression was barely detected. Using Pearson's $\chi^{2}$ test, it was demonstrated that CAP1 expression was associated with the histological grade and $\mathrm{Ki}-67$ expression. Kaplan-Meier analysis revealed that a higher CAP1 expression in patients with EOC was associated with a poorer prognosis. In in vitro experiments using HO-8910 EOC cells, the expression of CAP1 was knocked down using siRNA. The proliferation of the HO-8910 cells was then determined by cell cycle analysis and cell proliferation assay using the cell counting kit- 8 and flow cytometry. The results revealed that the loss of CAP1 expression inhibited cell cycle progression. These findings suggest that a high expression of CAP1 is involved in the pathogenesis of EOC, and that the downregulation of CAP1 in tumor cells may be a therapeutic target for the treatment of patients with EOC.
\end{abstract}

\section{Introduction}

Epithelial ovarian cancer (EOC) is one of the most common types of ovarian tumors and is the leading cause of mortality among

Correspondence to: Professor Jianxin Zhong, Department of Obstetrics and Gynecology, Affiliated Hospital of Nantong University, Xisi Road 20, Nantong, Jiangsu 226001, P.R. China

E-mail:ntzjx1965@126.com

*Contributed equally

Key words: adenylate cyclase-associated protein 1, human epithelial ovarian cancer, proliferation, therapeutic target gynecological malignancies $(1,2)$. Despite the rapid development of surgical and chemotherapy treatments, the 5-year survival rate for patients with EOC remains at approximately 30-50\% due to the lack of effective early diagnostic methods. Thus, there is an urgent need for the identification of biological factors with sensitivity and specificity in order to improve prognosis and reduce the threat to the lives and health of women.

It is well known that cell physiology, including cell-cell junctions, cytoskeletal structure and cell morphology regulate cancer cell migration and invasion. The actin cytoskeleton plays a key role in cancer cell motility and metastasis, as its remodeling regulates a number of important cellular processes, such as cell adhesion, migration and morphological changes (3-5). Cyclase-associated proteins (CAPs) which exist in organisms from mammals to apicomplexan parasites (6) are a family of evolutionarily conserved proteins which are key to regulating actin dynamics and participate in Ras-mediated adenylyl cyclase activity $(7,8)$. In mammal, cells have 2 CAP genes encoding the related CAP1 and CAP2 (9), both of which localize in the cell membrane and cytoplasm, and contain a C-CAP/co-factor C-like domain (10-13). The adenylate CAP1 gene, which encodes an actin monomer-binding protein is thought to facilitate processes, such as the establishment of cell polarity and mRNA localization (14). It also has the ability to control the cytoskeleton (15-19). The reorganization of the actin filament is regulated by actin-binding proteins in some signaling pathways that are essential for cell migration and several other intracellular events $(20,21)$. CAP1 also plays an important role in actin filament turnover by effectively recycling cofilin and actin (22). On both ends of the actin filament, CAP1 rapidly translocates to the mitochondria upon treatment with agents that induce apoptosis (23). Studies have demonstrated that CAP1 is overexpressed in hepatocelluar carcinoma (24), breast cancer (25), lung cancer (26) and esophageal squamous cell carcinoma (27). However, to the best of our knowledge, no more information is available to date regarding the role of CAP1 in ovarian tumorigenesis.

Therefore, in the present study, we aimed to investigate the expression and function of CAP1 in EOC. We analyzed the expression of CAP1 protein in 119 EOC tissue specimens by immunohistochemistry and western blot analysis. We also 
determined the correlation of CAP1 expression with clinicopathological characteristics and evaluated the prognostic value of CAP1 in EOC by survival analysis. In addition, we knocked down CAP1 expression in order to explore the potential involvement of CAP1 in the regulation of cell proliferation. Our results indicate that CAP1 is a newly identified biomarker for EOC and may be used as a therapeutic target for the treatment of EOC in the future.

\section{Materials and methods}

Patients and tissue samples. All investigations described in this study were carried out after obtaining informed consent and in accordance with an Institutional Review Board protocol approved by the Partners Human Research Committee at the Affiliated Hospital of Nantong University, Nantong, China. A total of 119 human EOC tissue specimens (45 papillary serous adenocarcinoma, 16 papillary mucinous carcinoma, 14 endometrioid adenocarcinoma and 14 clear cell carcinoma specimens, as well as 30 specimens classified as 'other') were provided by the Department of Pathology, the Affiliated Hospital of Nantong University from 2004 to 2009 . None of the patients had received chemotherapy or radiotherapy prior to surgery. A total of 10 tissue samples were obtained at the time of surgery and were immediately stored in liquid nitrogen and maintained at $-80^{\circ} \mathrm{C}$ until used in western blot analysis, including 1 normal tissue sample from a woman who underwent hysterectomy for benign disease. The clinicopathological characteristics of all the participants are shown in Table I. According to the WHO system, the histological classification of tumors was graded as follows: well differentiated [grade $1(\mathrm{G} 1) ; \mathrm{n}=15$ ], moderately differentiated [grade $2(\mathrm{G} 2) ; \mathrm{n}=33$ ] and poorly differentiated [grade $3(\mathrm{G} 3) ; \mathrm{n}=71$ ].

Antibodies. The antibodies used for western blot analysis and immunohistochemistry were as follows: mouse anti-CAP1 monoclonal antibody (SC-376286), mouse anti-human Ki-67 monoclonal antibody (SC-101861), mouse anti-human proliferating cell nuclear antigen (PCNA) monoclonal antibody (sc-56), rabbit anti-human cyclin A polyclonal antibody (sc-751) and rabbit anti-human glyceraldehyde 3-phosphate dehydrogenase (GAPDH) polyclonal antibody (sc-25778; all from Santa Cruz Biotechnology, Inc., Santa Cruz, CA, USA).

Western blot analysis. Total protein extracts (100 mg) were subjected to $10 \%$ sodium dodecyl sulfate-polyacrylamide gels electrophoresis (SDS-PAGE) and then transferred onto polyvinylidene difluoride (PVDF) filter membranes (Millipore, Bedford, MA, USA). After the membranes were blocked in $5 \%$ non-fat milk in TBST (150 mM NaCl, $20 \mathrm{mM}$ Tris, $0.05 \%$ Tween-20) for $2 \mathrm{~h}$, they were incubated with the primary antibodies overnight at $4^{\circ} \mathrm{C}$. After washing the membranes with TBST 3 times for $5 \mathrm{~min}$ each, horseradish peroxidase-linked IgG secondary antibodies (donkey anti-mouse IgG-HRP, sc-2314; Santa Cruz Biotechnology, Inc.) were added followed by incubation for $2 \mathrm{~h}$ at room temperature. The membranes were developed using the ECL detection system.

Immunohistochemistry. Serial sections (5- $\mu$ m-thick) were mounted on glass slides coated with $10 \%$ polylysine and were dewaxed in xylene and rehydrated in graded ethanol. Endogenous peroxidase activity was blocked by soaking in $0.3 \%$ hydrogen peroxide. The sections were then processed in $10 \mathrm{mmol} / 1$ citrate buffer (pH 6.0) and heated to $121^{\circ} \mathrm{C}$ in an autoclave for $20 \mathrm{~min}$ to retrieve the antigen. After rinsing in phosphate-buffered saline (PBS, pH 7.2), the sections were incubated with mouse anti-human CAP1 antibody (diluted 1:10,000) and mouse anti-human Ki-67 antibody (diluted 1:600) for $2 \mathrm{~h}$ at room temperature. Negative control slides were also processed in parallel using a non-specific immunoglobulin IgG (Santa Cruz Biotechnology, Inc.) at the same concentration as the primary antibody. All slides were processed using the peroxidase-anti-peroxidase method (Dako, Hamburg, Germany). Following rinsing with PBS, the peroxidase reaction was visualized by incubating the sections with the liquid mixture, DAB $(0.1 \%$ phosphate buffer solution, $0.02 \%$ diaminobenzidine tetrahydrochloride and $3 \% \mathrm{H}_{2} \mathrm{O}_{2}$ ). After rinsing in water, the sections were counterstained with hematoxylin, dehydrated and cover-slipped. All the immunostained sections were observed under a Leica fluorescence microscope (Leica Microsystems, Wetzlar, Germany).

For the assessment of CAP1 and Ki-67, at least 10 highpower fields in each specimen were randomly selected, and the percentage of cells with nuclear and cytoplasmic staining was examined with a total number of $>500$ cells counted to determine the labeling index in a single section. Tissues with no staining were rated as 0 , with a faint staining or moderate to strong staining in $\leq 25 \%$ of cells as 1 , with moderate staining or strong staining in $25-50 \%$ of cells as 2 , and strong staining in $\geq 50 \%$ of cells as 3 . For statistical analysis, a score of $<2$ was counted as low expression, while a score of $\geq 2$ was counted as overexpression, as previously described (24). When evaluating Ki-67 protein immunoreaction, staining was scored in a semiquantitative manner. A cut-off value of $50.7 \%$ or more positively stained nuclei in 5 high-power fields were used to identify Ki-67 staining as follows: the high expression group ( $\geq 50.7 \%)$ and low expression group $(<50.7 \%)$. All the immunostained sections were evaluated in a blinded manner without knowledge of the clinicopathological variables of the patients. In half of the samples, staining was repeated twice to avoid technical errors, but similar results were obtained in these samples.

Cell culture. The human EOC cells, HO-8910, were purchased from the Shanghai Institute of Cell Biology and cultured in RPMI-1640 supplemented with $10 \%$ heat-inactivated fetal calf serum and $100 \mathrm{U} / \mathrm{ml}$ penicillin-streptomycin mixture (both from Gibco-BRL, Grand Island, NY, USA) at $37^{\circ} \mathrm{C}$ and $5 \% \mathrm{CO}_{2}$.

Cell cycle analysis. Cells were harvested at the proper time and fixed in $70 \%$ ethanol overnight at $4^{\circ} \mathrm{C}$ and then incubated with $1 \mathrm{mg} / \mathrm{ml} \mathrm{RNase}$ A for $30 \mathrm{~min}$ at $37^{\circ} \mathrm{C}$. Subsequently, the cells were stained with propidium iodide (PI, $50 \mathrm{mg} / \mathrm{ml}$; BectonDickinson, San Jose, CA, USA) in PBS, 0.5\% Tween-20, and analyzed using a flow cytometer BD FACScan (BectonDickinson, San Jose, CA, USA) as well as CellQuest acquisition and analysis programs. As regards cell synchronization, serum deprivation was used for cell cycle G1-S phase arrest.

siRNA and transfection. Duplex siRNAs were synthesized as follows by Biomics Biotechnologies Co., Ltd. (Nantong, China): 
Table I. Expression of CAP1 in the 119 human ovarian cancer specimens.

\begin{tabular}{lccc}
\hline & \multicolumn{2}{c}{$\begin{array}{c}\text { CAP1 } \\
\text { expression }\end{array}$} \\
$\begin{array}{l}\text { Clinicopathological } \\
\text { characteristics }\end{array}$ & $\begin{array}{c}\text { No. of } \\
\text { cases }\end{array}$ & Low $\quad$ High & P-value \\
\hline
\end{tabular}

Age (years)
$\leq 50$
$>50$

FIGO stage, $\mathrm{n}(\%)$

I

II

III

IV

Histological grade, $\mathrm{n}(\%)$

1

2

3

Histological subtype, $\mathrm{n}(\%)$

Serous
Mucinous
Endometrioid
Clear cell
Others

Menopause

Absent

Present

Lymph node status, $\mathrm{n}(\%)$

Negative

Positive

Ascites, $\mathrm{n}(\%)$

Absent

Present

Malignant tumor cells in

peritoneal fluid, $\mathrm{n}(\%)$

$\begin{array}{llll}\text { Absent } & 90 & 41 & 49 \\ \text { Present } & 29 & 11 & 18\end{array}$

Metastases to other organs, $\mathrm{n}(\%)$

\begin{tabular}{lllll} 
Absent & 66 & 30 & 36 & \\
Present & 53 & 22 & 31 & \\
Ki-67 & & & & $<0.001^{\text {b }}$ \\
Low expression & 50 & 37 & 13 & \\
High expression & 69 & 15 & 54 & \\
\hline
\end{tabular}

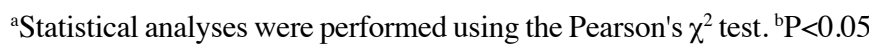
indicates statistical significance. CAP1, cyclase-associated protein 1.

The sequences were as follows CAP1-specific, 5'-GAAAUGAA UGAUGCCAdTdT-3' and control, 5'-UGGCGGCAUUCAUU UCdTdT-3'. The HO-8910 cells were seeded the day prior to transfection at a confluence of $50-70 \%$. In addition, a Mock group with no transfection was also used as a control. The transient transfection of CAP1 and non-specific siRNA oligos was carried out using Lipofectamine 2000 (Invitrogen, St. Louis, MO, USA) in accordance with the manufacturer's instructions. The cells were harvested $48 \mathrm{~h}$ after transfection and used for the experiment. The experiments were repeated at least 3 times.

Cell proliferation assay. The cell counting kit-8 (CCK-8) was used to measure cell proliferation according to the manufacturer's instructions (Dojindo, Kumamoto, Japan). The cells were seeded into a 96-well cell culture cluster plates (Corning, Inc., Corning, NY, USA) at a concentration of $2 \times 10^{4}$ cells/well in a volume of $100 \mu \mathrm{l}$ culture medium and grown overnight. CCK-8 reagents (Dojindo) were then added to each well followed by incubation for an additional $2 \mathrm{~h}$ at $37^{\circ} \mathrm{C}$. The absorbency was measured at a test wavelength of $490 \mathrm{~nm}$ and a reference wavelength of $630 \mathrm{~nm}$ using a microplate reader (Bio-Rad, Hercules, CA, USA). The experiments were repeated at least 3 times.

Statistical analysis. All statistical analyses were performed using SPSS 17.0 statistical software. The association between CAP1 and Ki-67 expression and clinicopathological characteristics was analyzed using Pearson's $\chi^{2}$ test. Multivariate analysis was performed using Cox's proportional hazards model. CAP1 and Ki-67 expression was quantified using Pearson's correlation co-efficient. Kaplan-Meier analysis was used to evaluate the survival curve, and the log-rank test was performed for analysis.

\section{Results}

CAPI is overexpressed in EOC tissues. To confirm the role of CAP1 in EOC, western blot analysis for CAP1 was performed using surgical specimens. We found that CAP1 protein expression gradually increased in 9 EOC tissues from 3 tumors classified as G1 to G3 (3 tissues from each tumor) in comparison with 1 normal ovarian tissue sample in which CAP1 expression was barely detected (Fig. 1). In order to determine the role of CAP1 in the progression and development of EOC, we examined the intracellular expression of CAP1 and Ki-67 in 119 specimens of EOC by immunohistochemical analysis. We found that CAP1 was mainly located in the cytoplasm of the EOC cells (Fig. 2). The high expression of CAP1 was accompanied by the high expression of $\mathrm{Ki}-67$ localized in the nucleus. In addition, CAP1 was highly expressed in the poorly differentiated tumor specimens compared to the well differentiated ones; similar results were obtained for Ki-67 expression.

Correlation of CAPI expression with clinicopathologic characteristics in EOC. To clarify the clinicopathological significance of CAP1, we analyzed the correlation of CAP1 expression with the clinicopathologic characteristics of patients with EOC (Table I). We divided the tumor specimens into 2 groups according to the expression of CAP1. CAP1 expression was significantly associated with the histological grade $(\mathrm{P}=0.010)$ and $\mathrm{Ki}-67$ expression $(\mathrm{P}<0.001)$, whereas there was no correlation observed with the FIGO stage $(\mathrm{P}=0.477)$, menopause $(\mathrm{P}=0.395)$, ascites $(\mathrm{P}=0.666)$, lymph node status $(\mathrm{P}=0.209)$, malignant tumor cells $(\mathrm{P}=0.472)$, metastases to other organs $(\mathrm{P}=0.666)$, age $(\mathrm{P}=0.775)$ and histologic subtype $(\mathrm{P}=0.071)$. In 
Table II. Survival status and clinicopathological characteristics in the 119 human ovarian cancer specimens.

\begin{tabular}{lc}
\hline $\begin{array}{l}\text { Clinicopathological } \\
\text { characteristics }\end{array}$ & Total \\
\cline { 1 - 1 } & Alive Deceased P-value
\end{tabular}

\begin{tabular}{llll}
\hline Age (years) & & & \\
$\leq 50$ & 35 & 15 & 20 \\
$>50$ & 84 & 23 & 61
\end{tabular}

FIGO stage, n (\%)

$\begin{array}{lrrr}\text { I } & 38 & 15 & 23 \\ \text { II } & 31 & 8 & 23 \\ \text { III } & 33 & 10 & 23 \\ \text { IV } & 17 & 5 & 12\end{array}$

Histological grade, n (\%)

$\begin{array}{rrrr}1 & 15 & 5 & 10 \\ 2 & 33 & 5 & 28 \\ 3 & 71 & 28 & 43\end{array}$

Histological subtype, n $(\%)$

Serous
Mucinous
Endometrioid
Clear cell
Others

Menopause

Absent

Present

Lymph node status, n (\%)

Negative

Positive

Ascites, n (\%)

Absent

Present

Malignant tumor cells in peritoneal fluid, n (\%)

$\begin{array}{lrrr}\text { Absent } & 90 & 32 & 58 \\ \text { Present } & 29 & 6 & 23\end{array}$

Metastases to other organs, $\mathrm{n}(\%)$

\begin{tabular}{lrrrr} 
Absent & 66 & 26 & 40 & \\
Present & 53 & 12 & 41 & \\
CAP1 & & & & $<0.001^{\mathrm{b}}$ \\
Low expression & 52 & 28 & 24 & \\
High expression & 67 & 10 & 57 & \\
Ki-67 & & & & $<0.001^{\mathrm{b}}$ \\
Low expression & 50 & 34 & 16 & \\
High expression & 69 & 4 & 65 & \\
\hline
\end{tabular}

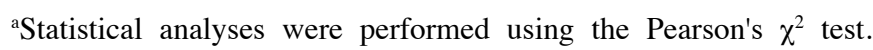
${ }^{\mathrm{b}} \mathrm{P}<0.05$ indicates statistical significance. CAP1, cyclase-associated protein 1 .

$0.047^{\mathrm{b}}$

0.081

0.051
Table III. Contribution of various potential prognostic factors to survival by Cox regression analysis of the 119 human ovarian cancer specimens.

\begin{tabular}{lcrc}
\hline & Hazard ratio & P-value & $95.0 \%$ CI \\
\hline Histological grade & 0.552 & $<0.001^{\mathrm{a}}$ & $0.396-0.770$ \\
CAP1 expression & 0.548 & $0.029^{\mathrm{a}}$ & $0.319-0.941$ \\
Ki-67 expression & 6.161 & $<0.001^{\mathrm{a}}$ & $3.256-11.655$ \\
\hline
\end{tabular}

${ }^{a} \mathrm{P}<0.05$ indicates statistical significance. CI, confidence interval; CAP1, cyclase-associated protein 1 .

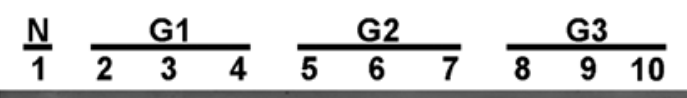

CAP1

GAPDH

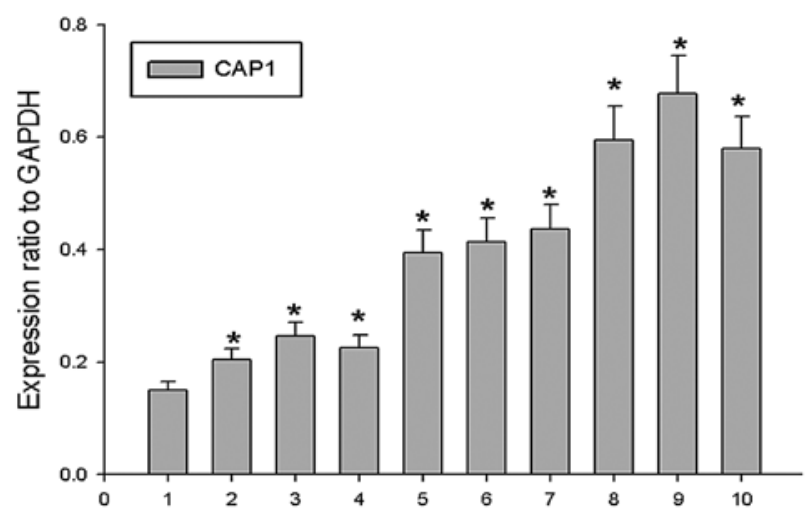

Figure 1. Cyclase-associated protein 1 (CAP1) is expressed in epithelial ovarian cancer (EOC) tissues. Expression of CAP1 in 1 normal tissue specimen $(\mathrm{N})$ and 9 cancer tissue specimens from 3 tumors classified as grade 1 (G1) to grade 3 (G3) (3 tissues from each tumor). GAPDH was used as a control for protein loading and integrity. The same experiment was repeated at least 3 times. ${ }^{*} \mathrm{P}<0.05$, compared with normal tissue.

addition, Pearson's correlation co-efficient revealed that there was a positive correlation between CAP1 expression and Ki-67 expression ( $\mathrm{r}=0.722, \mathrm{P}<0.001)$ (Fig. 3).

Expression of CAPI and prognosis of EOC. Kaplan-Meier analysis revealed that out of the 119 clinical cases examined, the patients with a high expression of CAP1 had a poorer overall survival than those with a lower expression $(\mathrm{P}<0.001$; Fig. 4). Pearson's $\chi^{2}$ test indicated that the histological grade $(\mathrm{P}=0.047)$, CAP1 $(\mathrm{P}<0.001)$ and Ki-67 $(\mathrm{P}<0.001)$ were independent prognostic indicators of EOC (Table II). Moreover, multivariate analysis using Cox's proportional hazards model showed that the histological grade $(\mathrm{P}<0.001), \mathrm{CAP} 1(\mathrm{P}=0.029)$ and $\mathrm{Ki}-67(\mathrm{P}<0.001)$ were independent prognostic indicators for patient overall survival (Table III). The survival curve indicated that the median survival for patients with a high CAP1 expression was significantly shorter than the median survival for those patients with a low CAP1 expression (Fig. 4). 

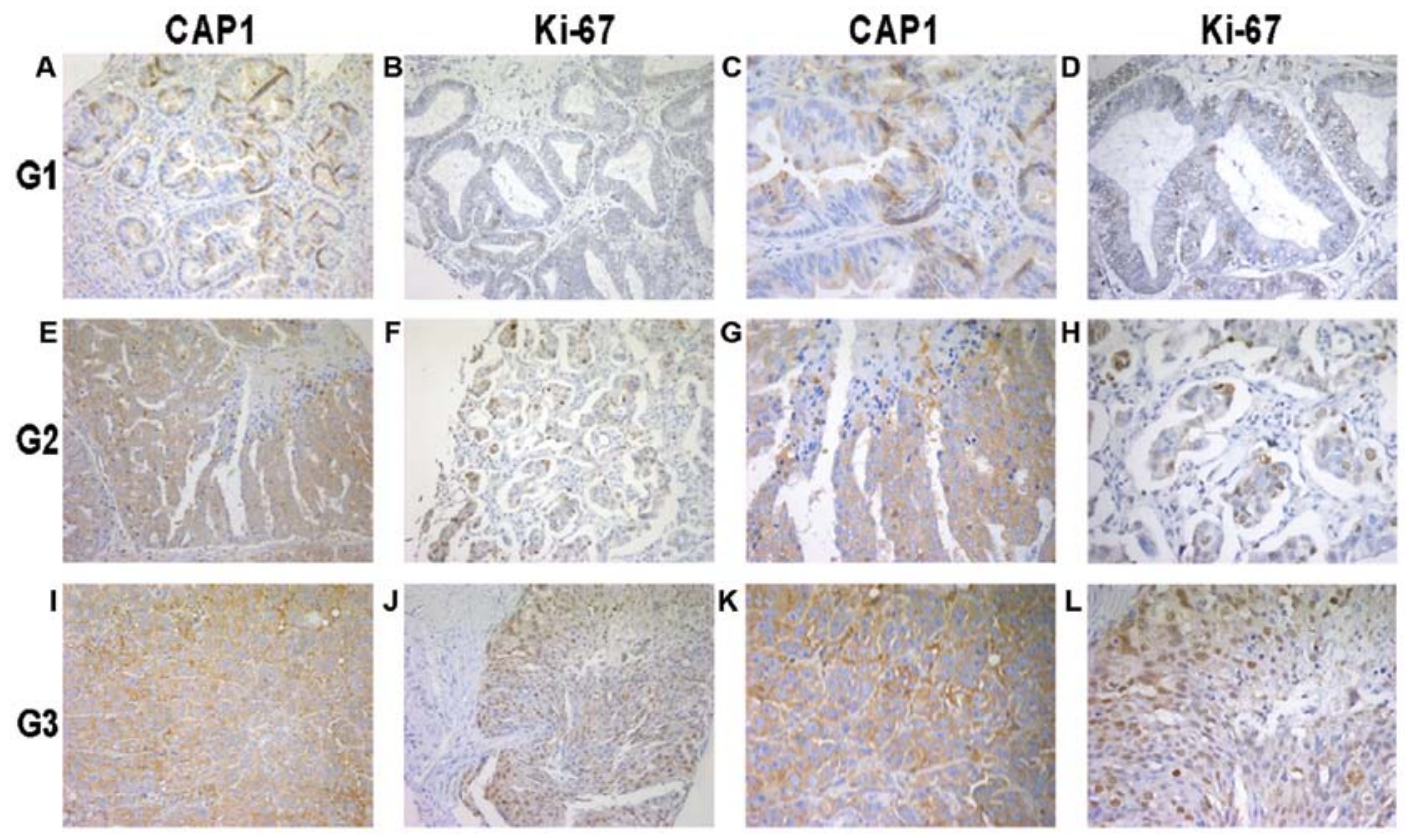

Figure 2. Immunohistochemical analysis of cyclase-associated protein 1 (CAP1) and Ki-67 expression in the 119 epithelial ovarian cancer (EOC) tissue specimens. Paraffin-embedded tissue sections were stained with antibodies to CAP1 and Ki-67 and counterstained with hematoxylin. (A-D) CAP1 and Ki-67 immunoreactivity in cancer tissue from a tumor classified as grade 1 (G1). (E-H) CAP1 and Ki-67 staining in cancer tissue from a tumor classified as grade 2 (G2). (I-L) CAP1 and Ki-67 staining in cancer tissue from a tumor classified as grade 3 (G3). The experiment details were described in 'Materials and methods'. (A, B, E, F, I and J) images at magnification, x200. (C, D, G, H, K and L) images at magnification, x400.

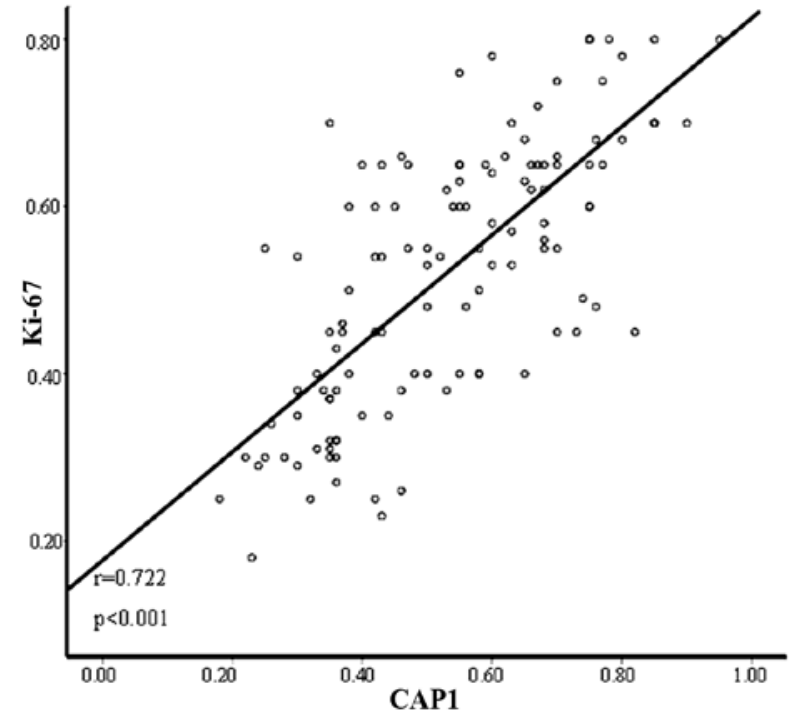

Figure 3. Correlation between cyclase-associated protein 1 (CAP1) and Ki-67 expression in epithelial ovarian cancer (EOC). Scatter plot of CAP1 against $\mathrm{Ki}-67$ with the regression line showing a correlation between them using Spearman's correlation co-efficient.

CAP1 expression and cell cycle progression. It has been reported that the knockdown of the expression of CAP1 affects the breast cancer cell cycle, inhibiting the growth of breast cancer cancer (25). In this study, we found that a high CAP1 expression associated with a poor prognosis of patients with EOC. Thus, we hypothesized that CAP1 participates in the cell cycle of HO-8910 cells. We subjected the cells to serum starvation and serum re-feeding and found that the expression of CAP1 gradually increased, as well as

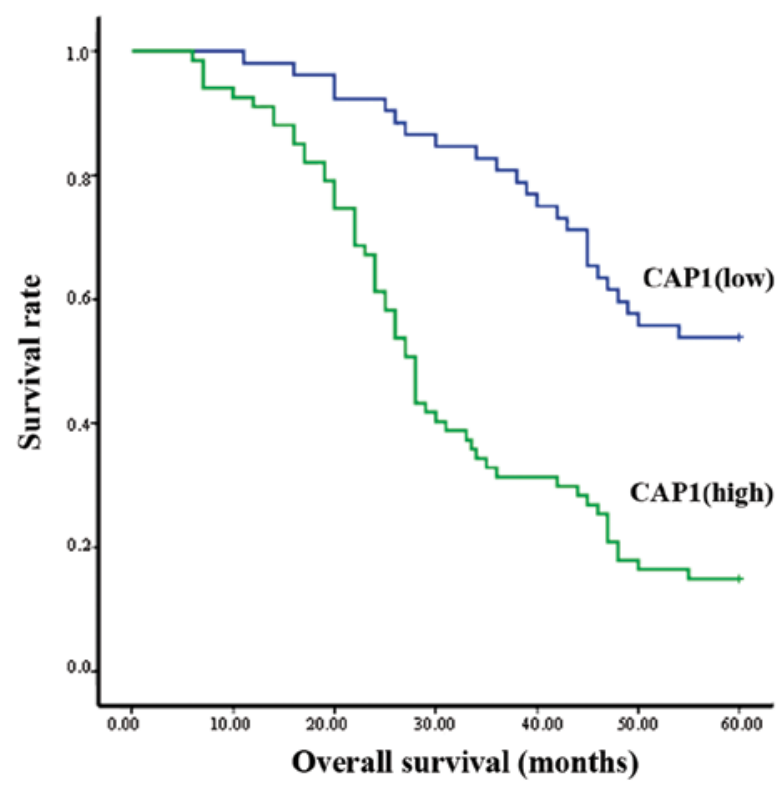

Figure 4. Kaplan-Meier survival curves demonstrate the correlation between survival and the expression of cyclase-associated protein 1 (CAP1) in 119 patients with epithelial ovarian cancer (EOC). Patients with a lower CAP1 expression had a longer survival than those with a higher CAP1 expression.

that of the proliferation markers, PCNA and cyclin A, during cell progression. Flow cytometric analysis revealed that the HO-8910 cells subjected to serum deprivation for $48 \mathrm{~h}$ were arrested in the G1 phase, and following serum re-feeding, the population of HO-8910 cells in the $\mathrm{S}$ phase increased from 18.08 to $61.12 \%$ (Fig. 5C). To further validate these results, we collected HO-8910 cellular protein at different 
A
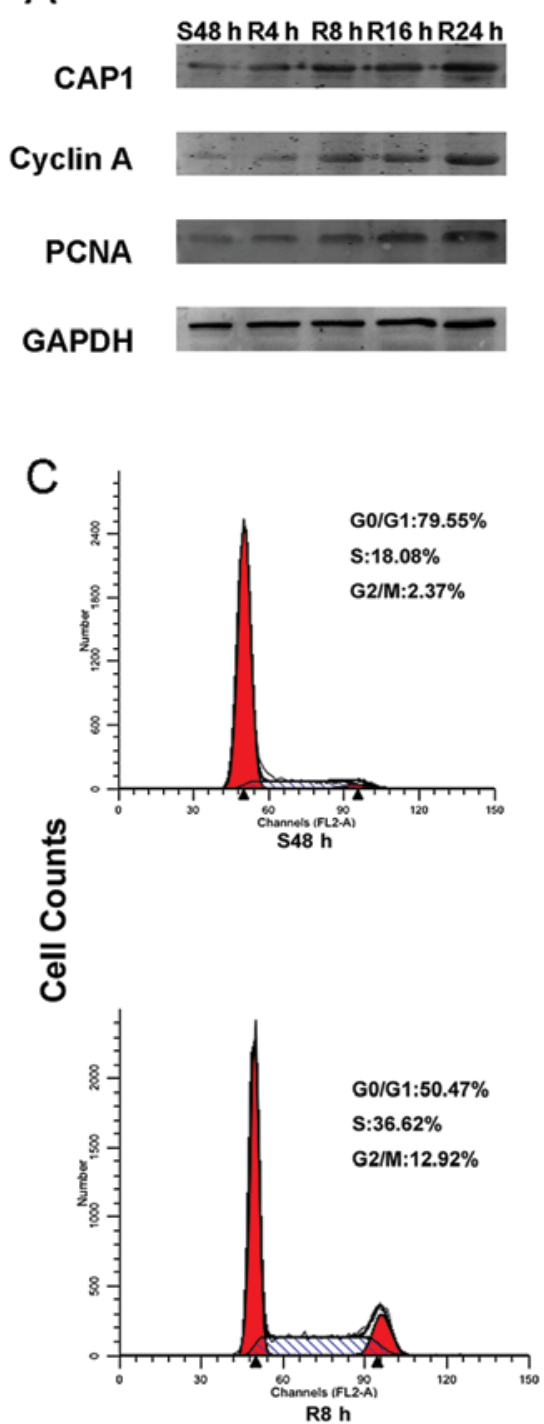
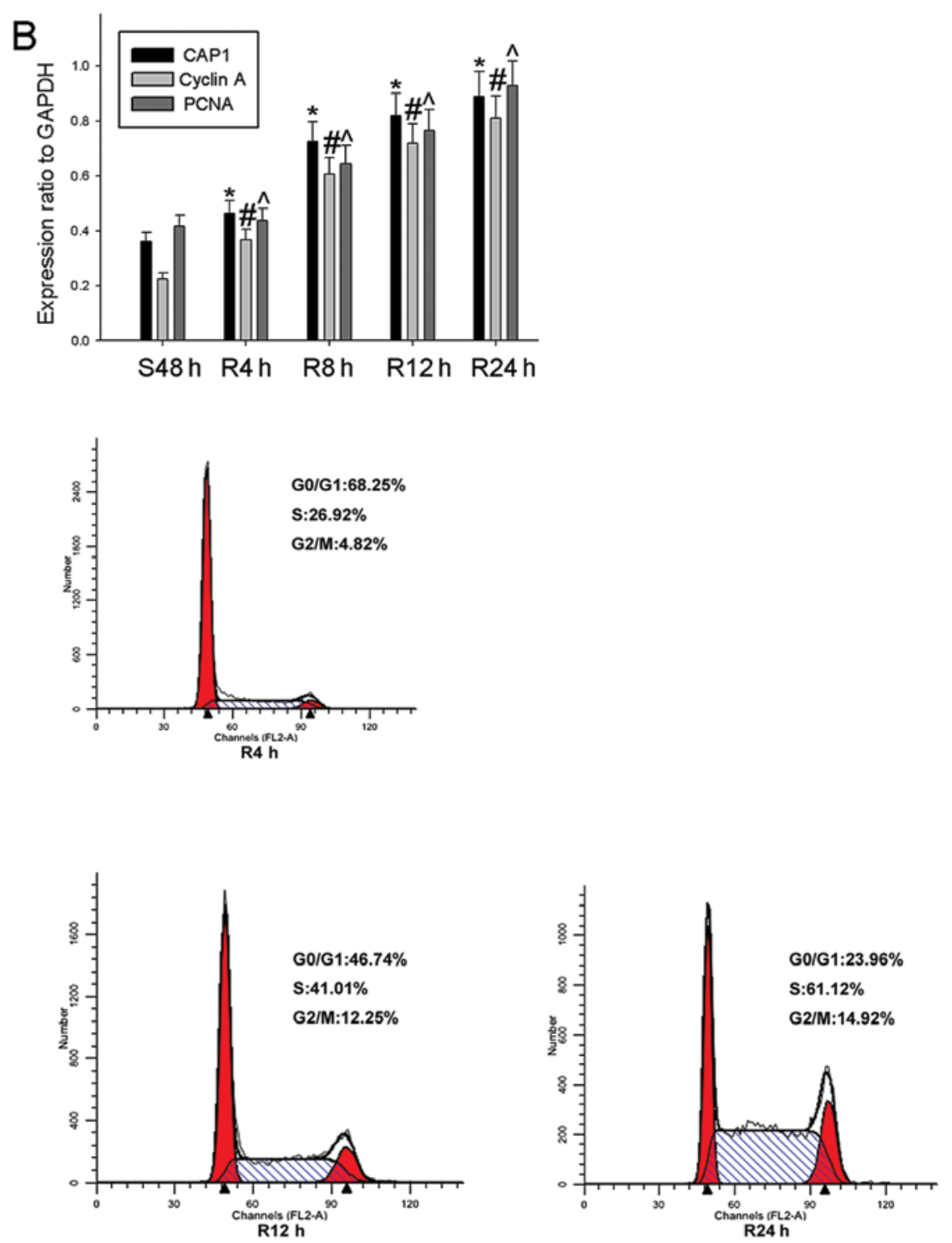

Figure 5. Expression of cyclase-associated protein 1 (CAP1) and cell cycle-related molecules in proliferating HO-8910 cells. (A) Cells were serum-starved for $48 \mathrm{~h}$ and following serum re-feeding, cell lysates were prepared and analyzed by western blot analysis using antibodies directed against CAP1, proliferating cell nuclear antigen (PCNA) and cyclin A. GAPDH was used as a control for protein loading and integrity. (B) The histogram demonstrates the ratio of CAP1, PCNA and cyclin A protein to GAPDH for each time point by densitometry. (C) Flow cytometric quantification of cell cycle progression in HO-8910. The cells were serum-starved for $48 \mathrm{~h}$, and medium containing $10 \%$ fetal bovine serum (FBS) was then added for the indicated periods of time ( $0,4,8,12$ and $24 \mathrm{~h})$. Data are the means \pm standard error of the mean (SEM) of 3 independent experiments. $n=3,{ }^{*, * \wedge} \mathrm{P}<0.05$, compared with control cells serum-starved for 48 h.

time points, and western blot analysis was performed to determine whether CAP1 expression is cell cycle-dependent in HO-8910 cells. We found that CAP1 expression was significantly increased as early as $4 \mathrm{~h}$ following serum re-feeding in the HO-8910 cells. The expression of the cell proliferation markers, PCNA and cyclin A, showed a similar tendency (Fig. 5A and B).

Knockdown of CAPl inhibits the proliferation of HO-8910 cells. To further determine the effect of CAP1 on cell proliferation, we knocked down endogenous CAP1 in HO-8910 cells using siRNA targeting CAP1 and the effects to the cells transfected with control siRNA and mock siRNA (Fig. 6A and B). The knockdown of CAP1 using siRNA inhibited the accumulation of cyclin A and PCNA compared with control- and mock-transfected cells (Fig. 6A and B). Additionally, we found that the proliferation rate of the HO-8910 cells transfected with siRNA exhibited a decrease compared with the cells transfected with the control siRNA and the mock-transfected cells (Fig. 6D). From these results, we ascertained that CAP1 plays a positive role in the regulation of cell proliferation.

\section{Discussion}

CAP1, a member of the CAP family in mammalian cells, was first identified as a component of the yeast adenylyl cyclase complex and conserved in all eukaryotic organisms. The CAP family contains 4 highly conserved protein domains, one of which is the N-terminal which interacts with adenylyl cyclase and induces the activity of RAS following exogenous signals, 

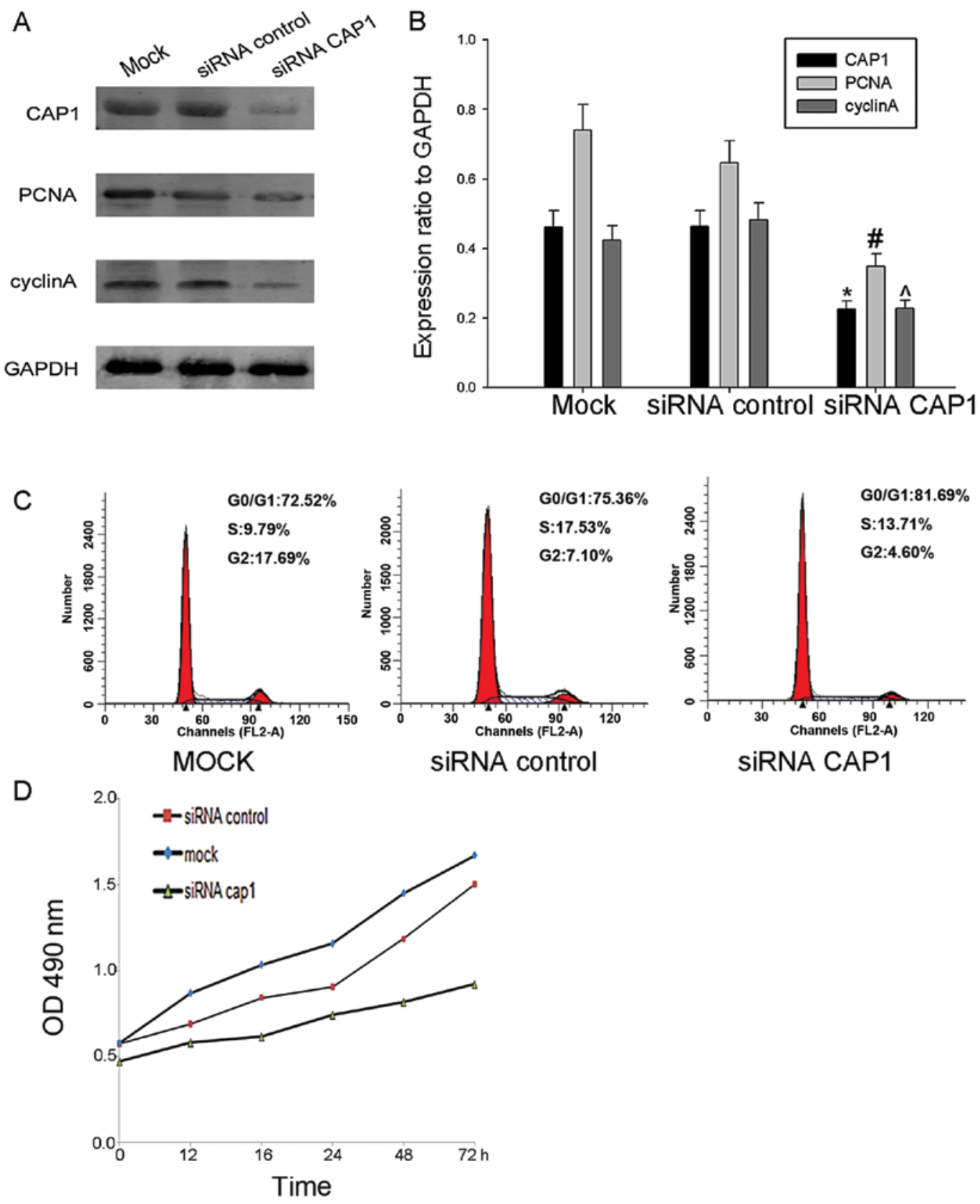

Figure 6. Knockdown of the expression of cyclase-associated protein 1 (CAP1) inhibits cell proliferation. (A) Western blot analysis was used to determine the levels of cyclin A and proliferating cell nuclear antigen (PCNA) in the HO-8910 cells following the depletion of CAP1. Transfection with siRNA led to a decrease in CAP1 expression, as well as in PCNA and cyclin A expression, as shown by western blot analysis $48 \mathrm{~h}$ following the transfection of HO-8910 cells. (B) Histogram demonstrates the ratio of CAP1, PCNA and cyclin A protein to GAPDH at each time point by densitometry. (C) Cell cycle analysis following the downregulation of CAP1 by siRNA targeting CAP1 in HO-8910 cells. Following transfection of the HO-8910 cells with siRNA targeting CAP1, an increase in the cell number at the G0/G1 phase was observed, with a concomitant decrease in the number of cells in the S phase, as shown by flow cytometry. (D) Comparison of growth curves between the cells transfected with siRNA targeting CAP1 and control siRNA and mock-transfected cells using the cell counting kit-8 (CCK-8). The data are the means \pm standard error of the mean (SEM) $\left(\mathrm{n}=3 ;{ }^{* * *, *} \mathrm{P}<0.05\right.$, compared with the control- and mock-transfected cells). Each experiment was repeated at least 3 times.

whereas the C-terminal half of CAPs is involved in the cell differentiation and depolymerization of the F-actin filamentactin (28). As a monomeric actin binding protein, CAP is involved in cell polarization, the distribution of actin filaments and mRNA in a Dictyostelium (29). The expression of CAP is associated with an abnormally large cell size, random budding pattern and an abnormal actin distribution in yeast $(30,31)$.

A number of scholars have started to investigate the association between CAP1 and cancer. CAP1 has been to be commonly overexpressed in pancreatic cancers, and its level in clinical cases has been shown to be associated with neuronal invasion and lymph node metastasis. The knockdown CAP1 has been shown to reduce cell motility and migration (32). It has been reported that CAP1 is upregulated in breast cancer. After knocking down its expression, the proliferation and migration of MDA-MB-231 cells was shown to decrease, inducing changes in morphology, which were associated with the arrangement of F-actin (25). Western blot 
analysis, real-time PCR and immunohistochemical analysis have been used to prove that CAP1 is overexpressed in hepatocellular carcinoma compared with adjacent non-cancerous liver tissues, and that it is positively associated with HCC cell metastasis (24). CAP1 overexpression has been shown to be significantly associated with lymph node status in esophageal squamous cell carcinoma. The knockdown of CAP1 in TE1 cells has been shown to result in a decreased migration capability and the overexpression of CAP1 promotes TE1 cell migration (27). In this study, we found that CAP1 was overexpressed in EOC. Using immunohistochemistry, we found that CAP1 was highly expressed in poorly differentiated specimens compared to well differentiated ones, and similar results were obtained for Ki-67 expression. KaplanMeier analysis revealed that out of the 119 clinical cases, the patients with a high expression of CAP1 had a poorer overall survival than those with a lower CAP1 expression. In addition, the knockdown of CAP1 expression in an in vitro experiment revealed that the loss of CAP1 inhibit the proliferation of HO-8910 cells.

It has been demonstrated that the morphologic changes of malignant tumor cells enhance the migration capacity of the cells and lead to invasion and metastasis (33). To a certain degree, the maintainance and changes in the structure and function of cells are achieved by regulating the structure and function of the actin cytoskeleton, which is the key to the reorganization of the actin cytoskeleton. When stimulated, CAP regulates the polymerization and disassembly of downstream actin protein, thus affecting the growth and differentiation of cells. CAP1 also takes part in accelerating the turnover of actin filaments by the recycling of cofilin and actin on both ends of the actin filament (34). Therefore, we hyopthesized that the molecular mechanisms of action of CAP1 in the pathogenesis of EOC may involve its downstream actin protein. However, further studies are required to identify the precise signaling pathways involved.

As CAP1 is overexpressed in EOC, it may thus serve as a prognostic marker for EOC. Using western blot analysis, we found that the expression of CAP1 was higher in the 9 EOC tissues compared to the 1 normal tissue, in which CAP1 expression was barely detected. Furthermore, immunohistochemistry of the 119 paraffin-embedded tissue sections of EOC also revealed that CAP1 immunostaining was located in the cytoplasm and was upregulated in the poorly differentiated tumor cells compared to the well differentiated ones. These results were similar to those obtained for $\mathrm{Ki}-67$ expression, highlighting that CAP1 expression significantly correlated with the proliferation of EOC cells. The association between CAP1 and markers of the cell cycle was then detected by a starvation-release experiment.

In conclusion, this study demonstrates that CAP1 is an independent prognostic factor in EOC. Our data suggest that CAP1 plays an important role in cell proliferation in EOC and that CAP1 may be a potential therapeutic target in EOC chemotherapy.

\section{Acknowledgements}

This study was supported by a grant from the National Natural Science Foundation of China (no. 81302285).

\section{References}

1. Menczer J, Chetrit A, Sadetzki S; National Israel Ovarian Cancer Group: Uterine metastases in ovarian carcinoma: frequency and survival in women who underwent hysterectomy. J Gynecol Oncol 21: 191-195, 2010.

2. Park B, Park S, Kim TJ, et al: Epidemiological characteristics of ovarian cancer in Korea. J Gynecol Oncol 21: 241-247, 2010.

3. Chang E, Heo KS, Woo CH, et al: MK2 SUMOylation regulates actin filament remodeling and subsequent migration in endothelial cells by inhibiting MK2 kinase and HSP27 phosphorylation. Blood 117: 2527-2537, 2011.

4. Kirfel G, Rigort A, Borm B and Herzog V: Cell migration: mechanisms of rear detachment and the formation of migration tracks. Eur J Cell Biol 83: 717-724, 2004.

5. Hall A: The cytoskeleton and cancer. Cancer Metastasis Rev 28: 5-14, 2009.

6. Makkonen M, Bertling E, Chebotareva NA, Baum J and Lappalainen P: Mammalian and malaria parasite cyclase-associated proteins catalyze nucleotide exchange on G-actin through a conserved mechanism. J Biol Chem 288: 984-994, 2013.

7. Fedor-Chaiken M, Deschenes RJ and Broach JR: SRV2, a gene required for RAS activation of adenylate cyclase in yeast. Cell 61: 329-340, 1990.

8. Field J, Vojtek A, Ballester R, et al: Cloning and characterization of CAP, the S. cerevisiae gene encoding the $70 \mathrm{kd}$ adenylyl cyclase-associated protein. Cell 61: 319-327, 1990.

9. Peche V, Shekar S, Leichter M, et al: CAP2, cyclase-associated protein 2, is a dual compartment protein. Cell Mol Life Sci 64: 2702-2715, 2007.

10. Matviw H, Yu G and Young D: Identification of a human cDNA encoding a protein that is structurally and functionally related to the yeast adenylyl cyclase-associated CAP proteins. Mol Cell Biol 12: 5033-5040, 1992.

11. Zelicof A, Gatica J and Gerst JE: Molecular cloning and characterization of a rat homolog of CAP, the adenylyl cyclaseassociated protein from Saccharomyces cerevisiae. J Biol Chem 268: 13448-13453, 1993.

12. Yu G, Swiston J and Young D: Comparison of human CAP and CAP2, homologs of the yeast adenylyl cyclase-associated proteins. J Cell Sci 107: 1671-1678, 1994.

13. Swiston J, Hubberstey A, Yu G and Young D: Differential expression of CAP and CAP2 in adult rat tissues. Gene 165: 273-277, 1995.

14. Pollard TD and Cooper JA: Actin, a central player in cell shape and movement. Science 326: 1208-1212, 2009.

15. Baum B, Li W and Perrimon N: A cyclase-associated protein regulates actin and cell polarity during Drosophila oogenesis and in yeast. Curr Biol 10: 964-973, 2000.

16. Baum B and Perrimon N: Spatial control of the actin cytoskeleton in Drosophila epithelial cells. Nat Cell Biol 3: 883-890, 2001.

17. Hubberstey AV and Mottillo EP: Cyclase-associated proteins: CAPacity for linking signal transduction and actin polymerization. FASEB J 16: 487-499, 2002.

18. Kang CM and Jiang YW: Genome-wide survey of non-essential genes required for slowed DNA synthesis-induced filamentous growth in yeast. Yeast 22: 79-90, 2005.

19. Yusof AM, Hu NJ, Wlodawer A and Hofmann A: Structural evidence for variable oligomerization of the N-terminal domain of cyclase-associated protein (CAP). Proteins 58: 255-262, 2005.

20. Loisel TP, Boujemaa R, Pantaloni D and Carlier MF: Reconstitution of actin-based motility of Listeria and Shigella using pure proteins. Nature 401: 613-616, 1999.

21. Pollard TD, Blanchoin L and Mullins RD: Molecular mechanisms controlling actin filament dynamics in nonmuscle cells. Annu Rev Biophys Biomol Struct 29: 545-576, 2000.

22. Bertling E, Hotulainen P, Mattila PK, Matilainen T, Salminen M and Lappalainen P: Cyclase-associated protein 1 (CAP1) promotes cofilin-induced actin dynamics in mammalian nonmuscle cells. Mol Biol Cell 15: 2324-2334, 2004.

23. Wang C, Zhou GL, Vedantam S, Li P and Field J: Mitochondrial shuttling of CAP1 promotes actin- and cofilin-dependent apoptosis. J Cell Sci 121: 2913-2920, 2008.

24. Liu Y, Cui X, Hu B, et al: Upregulated expression of CAP1 is associated with tumor migration and metastasis in hepatocellular carcinoma. Pathol Res Pract 210: 169-175, 2014.

25. Yu XF, Ni QC, Chen JP, et al: Knocking down the expression of adenylate cyclase-associated protein 1 inhibits the proliferation and migration of breast cancer cells. Exp Mol Pathol 96: 188-194, 2014. 
26. Tan M, Song X, Zhang G, et al: Overexpression of adenylate cyclase-associated protein 1 is associated with metastasis of lung cancer. Oncol Rep 30: 1639-1644, 2013.

27. Li M, Yang X, Shi H, et al: Downregulated expression of the cyclase-associated protein 1 (CAP1) reduces migration in esophageal squamous cell carcinoma. Jpn J Clin Oncol 43: 856-864, 2013.

28. Zelicof A, Protopopov V, David D, Lin XY, Lustgarten V and Gerst JE: Two separate functions are encoded by the carboxylterminal domains of the yeast cyclase-associated protein and its mammalian homologs. Dimerization and actin binding. J Biol Chem 271: 18243-18252, 1996.

29. Noegel AA, Blau-Wasser R, Sultana H, et al: The cyclaseassociated protein CAP as regulator of cell polarity and cAMP signaling in Dictyostelium. Mol Biol Cell 15: 934-945, 2004.
30. Gerst JE, Ferguson K, Vojtek A, Wigler M and Field J: CAP is a bifunctional component of the Saccharomyces cerevisiae adenylyl cyclase complex. Mol Cell Biol 11: 1248-1257, 1991.

31. Vojtek A, Haarer B, Field J, et al: Evidence for a functional link between profilin and CAP in the yeast $S$. cerevisiae. Cell 66 : 497-505, 1991.

32. Yamazaki K, Takamura M, Masugi Y, et al: Adenylate cyclaseassociated protein 1 overexpressed in pancreatic cancers is involved in cancer cell motility. Lab Invest 89: 425-432, 2009.

33. Friedl $\mathrm{P}$ and Wolf $\mathrm{K}$ : Tumour-cell invasion and migration: diversity and escape mechanisms. Nat Rev Cancer 3: 362-374, 2003.

34. Moriyama K and Yahara I: Human CAP1 is a key factor in the recycling of cofilin and actin for rapid actin turnover. J Cell Sci 115: 1591-1601, 2002. 